Results Out of the 30 infants born to syphilis seropositive mothers, we found 3 babies with different IgG WB profiles from those of their own mothers. Two out these three newborns had also positive IgM WB result. Routine serological testing results of all the 30 newborns showed similar values to those of their own mothers.

Conclusion The use of comparative IgG WB test enabled us to diagnose CS in three cases in which the infection would have not been detected by classical serology techniques. Therefore the routine use of comparative IgG WB assay at birth on newborn-mother pairs could be a welcome addition to the conventional laboratory methods used for the diagnosis of CS.

\section{P3.351 CONGENITAL SYPHILIS PREVENTION IN HONDURAS}

doi:10.1136/sextrans-2013-051184.0804

M A U B Urquia. Secretaria de Salud, Tegucigalpa, Honduras

Introduction Honduras is a Central American country whose priority is the elimination of congenital syphilis in 2015.

Objective To prevent transplacental transmission of syphilis from mother to daughter/son during the gestational period.

Materials and Methods Descriptive retrospective analysis from January 2008 to December 2012 in 117 health units without a laboratory from the Preventive Program transmission of syphilis from mother to daughter/son that detects syphilis during pregnancy in attending antenatal women, using rapid tests for free and voluntary screening.

Results Year 20084.950 pregnant women were screened, 10 of them were reactive, with a prevalence of $0.2 \%$, in 20097.285 pregnant women were screened of which 17 were reactive with a prevalence of $0.2 \%$, in the year 20105.310 pregnant women were screened, 5 out were reactive with a prevalence of $0.09 \%$, in 2011 8.343 pregnant women were screened of which 20 were reactive with prevalence of $0.2 \%$ and 20129.320 women were screened of which 14 were reactive with a prevalence of $0.1 \%$.

Conclusions During the five years 66 pregnant women were screened with syphilis, all received treatment with their partners and were followed until delivery, of which 57 (86\%) of the children born were free of congenital syphilis, 7 pregnant women have not yet reached the time birth and 1 pregnant woman did not return for follow-up service.

Screening for syphilis during pregnancy is key to preventing transplacental transmission of syphilis mother daughter/son

The free screening with rapid tests for syphilis in pregnant women in places that do not have clinical laboratories, improved the access and permitted the early capture, and were provided with timely treatment with their sexual partner.

Rapid tests for syphilis are essential tool for early diagnosis of congenital syphilis prevention.

\section{P3.352 PREVENTION OF CONGENITAL SYPHILIS}

doi:10.1136/sextrans-2013-051184.0805

N Potekaev, N Zatorskaya, 0 Porshina. Moscow Scientific-Research Center of Dermatovenerology and Cosmetology, Moscow, Russian Federation

Congenital syphilis in Russia is an actual problem. The number of children with this disease was respectively 2005-325, 2006-321, 2007325, 2008-249, 2009-190, 2010-175 persons. The source of the high morbidity are pregnant women with syphilis, who didn't know about their disease before childbirth. The aim is to evaluate the importance of prevention of syphilis that is possible with the deepest analysis of health of each child. Complex inspections of 51 children with early congenital syphilis revealed that $84.3 \%$ had manifested forms.

At birth $62.7 \%$ had Apgar score below $7 / 8$ points, $25.4 \%-7 / 8$ points, $11.8 \%$ - above $7 / 8$ points. While in the hospital $5.9 \%$ - had satisfactory status, $13.7 \%$ - moderately grave condition, $80.3 \%$ critical condition, among them $54.9 \%$ - premature babies.

Clinical signs of early congenital syphilis symptoms were bone changes $-66.7 \%$. In $19.6 \%$ of cases, the bone changes were the only specific clinical signs. The skin and mucous membranes observed in $37.3 \%$ : syphilitic pemphigus $-27.5 \%$, infiltration of the palms and soles with desquamation krupnoplastinchatym - 7.8\%, roseola $5.9 \%$, rhinitis $-7.8 \%$.

Changes in the internal organs was found in $68.6 \%$, hepatomegaly $-60.8 \%$, hepatitis $-9.8 \%$, splenomegaly - $39.2 \%$, myocarditis $2 \%$, pneumonia $-5.9 \%$ and nephritis- $39 \%$.

Damage of nervous system (specific meningitis) registered at $23.5 \%$ that is every 4 th child.

All children had a hypoxic-ischemic damage of central nervous system with syndromes of depression and agitation, psychomotor retardation, perinatal encephalopathy.

Almost all of them had immaturity of the functional-and-morphological systems (lengthening period of neonatal jaundice, syndrome of respiratory disorders and nervous regulation).

Thus the syphilis infection in pregnancy increases the risk of developing specific and non-specific disorders in formation of bodies and systems of a foetus.

Moscow Center uses obtained analytical data for planning preventive measures in this target group.

\section{P3.353 CONGENITAL SYPHILIS IN BUENAVENTURA, COLOMBIA: DESCRIPTION OF A CASE SERIES IN A NEGLECTED PACIFIC COAST TOWN}

doi:10.1136/sextrans-2013-051184.0806

${ }^{1} \mathrm{M}$ A Castrillon, ${ }^{1}$ A R Cruz, ${ }^{2} Y$ Minota, $1,3 \mathrm{~L}$ C Rubiano, ${ }^{3} \mathrm{M}$ C Castaño, ${ }^{1} \mathrm{M}$ Perez, 4.5,1 J C Salazar. ' CIDEIM, Cali, Colombia; ${ }^{2}$ Hospital Departamental de Buenaventura, Buenaventura, Colombia; ${ }^{3}$ Secretaria de Salud Departamental del Valle, Cali, Colombia; ${ }^{4}$ University of Connecticut Health Center, Farmington, CT, United States; ${ }^{5}$ Connecticut Children's Medical Center, Hartford, CT, United States

Background Syphilis in pregnant women is a world-wide health problem leading to serious adverse outcomes. Although there are strategies to prevent congenital syphilis (CS) through antenatal screening, disease burden still remains high especially in lowresourced settings. Buenaventura, a municipality in the Colombian Pacific-Coast, is endemic for gestational syphilis (GS) and accounts for the $6.6 \%$ of CS in Colombia. This study describes the CS problem in Buenaventura, giving rise to strategies that could generate positive impact in prevention of syphilis mother-to-child transmission.

Methods A retrospective health record review of CS patients and mothers that attended to the Buenaventura Hospital during a 7-month period, 2011, was conducted. Demographic, antenatal care (ANC), syphilis diagnosis/treatment information from GS patients, and clinical information from CS cases was collected. A descriptive analysis of the information was performed, analysed using SSPS 20.0. Study procedures were approved by CIDEIM's human subject's board.

Results 100 clinical records were reviewed; 89 and 92 cases met definitions for CS and GS respectively. All GS mothers lived in the municipality of Buenaventura, mainly in urban areas. The majority $(80 \%)$ were affiliated to private health-care providers. Although $70 \%$ of GS mothers attended to at least one ANC visit, and $64 \%$ had at least one VDRL test, only $10 \%$ received adequate antibiotic therapy, and $2 \%$ of the partners were treated. Stillbirths and early neonatal deaths $(7 / 82)$, preterm births $(15 / 82)$ were the main finding in the CS population. All infants were hospitalised for intravenous antibiotic treatment.

Conclusions The high CS incidence in Buenaventura (7/1000 live-births), is the result of lack of appropriate ANC, which ultimately leads to failures in syphilis screening and adequate treatment. CS prevention strategies should be focused in early syphilis 